\title{
A Multistage, Multimethod Approach for Automatic Detection and Classification of Epileptiform EEG
}

\author{
He Sheng Liu*, Tong Zhang, and Fu Sheng Yang
}

\begin{abstract}
An efficient system for detection of epileptic activity in ambulatory electroencephalogram (EEG) must be sensitive to abnormalities while keeping the false-detection rate to a low level. Such requirements could be fulfilled neither by single stage nor by simple method strategy, due to the extreme variety of EEG morphologies and frequency of artifacts. The present study proposes a robust system that combines multiple signal-processing methods in a multistage scheme, integrating adaptive filtering, wavelet transform, artificial neural network, and expert system. The system consists of two main stages: a preliminary screening stage in which data are reduced significantly, followed by an analytical stage. Unlike most systems that merely focus on sharp transients, our system also takes into account slow waves. A nonlinear filter for separation of nonstationary and stationary EEG components is also developed in this paper. The system was evaluated on testing data from 81 patients, totaling more than 800 hours of recordings. $90.0 \%$ of the epileptic events were correctly detected. The detection rate of sharp transients was $98.0 \%$ and overall false-detection rate was $6.1 \%$. We conclude that our system has good performance in detecting epileptiform activities and the multistage multimethod approach is an appropriate way of solving this problem.
\end{abstract}

Index Terms-Adaptive filtering, artificial neural network, electroencephalogram (EEG), epilepsy, wavelet transform.

\section{INTRODUCTION}

$\mathbf{L}$ ONG-TERM electroencephalogram (EEG) recording is a widely used clinical procedure for the diagnosis of epilepsy because it is more likely to capture epileptiform abnormalities, both ictal and interictal, than short-term recording. Ambulatory EEG (AEEG), which allows the patients to move with the portable apparatus while the EEG data are recorded continuously, has proven to be particularly useful for the following purposes: 1) to confirm a clinical suspicion of epilepsy; 2) to identify interictal epileptiform activity; 3 ) to document seizures that the patient is unaware of; 4) to evaluate response to therapy; 5) to evaluate nocturnal or sleep-related events; 6) to evaluate suspected pseudoseizures; and 7) to evaluate syncope [1].

However, human visual review of the vast amount of AEEG data has serious drawbacks. Visual inspection is prohibitively

Manuscript received January 16, 2001; revised November 8, 2002. This work was supported by the National Natural Science Foundation of China under Grant 59937160. Asterisk indicates corresponding author.

${ }^{*} \mathrm{H}$. S. Liu is with the Institute of Biomedical Engineering, Department of Electrical Engineering, Tsinghua University, Beijing 100084, China (e-mail: heshengliu@yahoo.com).

T. Zhang and F. S. Yang are with the Institute of Biomedical Engineering, Department of Electrical Engineering, Tsinghua University, Beijing 100084 China

Digital Object Identifier 10.1109/TBME.2002.805477 time-consuming and inefficient. As AEEG recording can last for a whole week, even if the EEGer reads the data ten times faster than the recording speed, reviewing a dataset will take more than $16 \mathrm{~h}$. Obviously, such exhausting work will overburden the reader and the detection accuracy could be undermined when the reader becomes tired. Visual inspection lacks standards. Although most EEGers tend to have identical overall conclusions on an EEG dataset, they may diverge in interpretation of specific events. Even the same reader may judge the same event differently at different times. Visual inspection lacks quantitative analysis which can uncover hidden characters of the data [2], [3].

Thus computer-assisted analysis becomes quite necessary in practice. To date, many automated detection algorithms have been developed. They can be roughly divided into the following categories.

a) Orthogonal transform: These methods, such as fast Fourier transform (FFT), capture the rhythmic change of EEG. Some researchers have used this method to detect the $3 \mathrm{c} / \mathrm{s}$ spike-and-wave complex [4]. Nevertheless, orthogonal transform must average a data segment, hence, losing temporal details.

b) Template matching: Templates of epileptiform waves are set. When the cross correlation between a template and an EEG wave exceeds a threshold, an alarm signals epileptiform activity [5]. Due to the variety of epileptiform waves, defining a set of templates that are suitable for all cases is difficult. Furthermore, compromise between false detection and omission is hard to make when setting the threshold.

c) Inverse filtering: Normal EEG is assumed to be the output of a filter that has fixed parameters while the input is white noise. When EEG data pass through the inverse filter, if the output is white noise, then the input is considered to be stationary, otherwise it is considered nonstationary and an alarm is triggered [5], [6]. The pitfall of this method is that false detection becomes serious when nonstationary artifacts occur. Further, if the characteristics of EEG changes with time but the parameters remain fixed, then the filter's efficiency will fade.

d) Expert system: This method mimics human visual inspection and is considered to be a promising method. Parameters of EEG wave (such as amplitude, width, slope, etc.) are calculated and thresholded [7]-[9]; at the same time, some additional information (temporal and spatial contexts, status of the subject, etc.) is integrated to detect epileptic waves and reject artifacts [10]-[13]. 
e) Artificial neural network (ANN): The raw EEG or some parameters of the EEG wave are weighted and combined to form a criterion. Then, output of the criterion is compared with the threshold derived from previous training [14]-[18].

f) Wavelet transform: It is a new approach of time-frequency analysis and its advantage in epileptiform EEG detection has been reported by a great number of researchers [19]-[21].

g) Other methods: Fuzzy clustering [22], chaos theory [23], and various other methods have been reported [24], [25].

Each method has some unique advantages in signal processing, but none of them alone can fulfill the requirement of epileptic EEG detection. Now it is widely recognized that a promising way to solve such a complicated problem is to combine these methods and let them supplement each other. Jones et al. [8] have proposed a multistage system that integrated a feature extractor and expert system together. They also claimed that the only way to separate epileptiform from nonepileptiform wave is to make use of a wide spatial and temporal context. Gotman et al. [26] developed three methods separately and combined the results together to achieve a higher detection rate, but they also got higher false-detection rate at the same time.

Epileptic EEG is characterized by certain abnormalities, including spikes, sharp waves, slow waves and some slow-wavebased complexes (spike-and-wave complex or sharp-and-wave complex). However, most epilepsy detection algorithms in the literature are based on the identification of sharp transients such as spike and sharp wave [2]-[4], [8], [11], [17], [18], [20]. This is quite insufficient because during the ictal period the EEG often appears to be rhythmic and slow waves become an important sign in such circumstances. Although interictal spikes provide evidence of epilepsy, interictal slow waves can also play an important role for the diagnosis, especially in many cases when the sharp transients do not appear. Gotman et al. [26] have noticed the importance of slow waves in seizure detection and have employed a method to detect slow rhythmic discharges in their system. Unfortunately, identification of slow waves is far from direct because their morphology varies more greatly than that of sharp transients. The difficulty of detection may be one of the reasons why slow waves have not been fully used. Nevertheless, the ignorance of the slow waves in most automatic detection systems has hindered their efficient use in clinics.

In order to maintain high sensitivity and low false-detection rate for all types of epileptic waves, we propose a system based on a hierarchical multimethod approach, which integrates the information embedded in the time, space and frequency domains. The combination of multiple methods makes the system robust and reliable.

Fig. 1 shows the block diagram of the system, which consists of two main stages. 1) The preliminary screening stage picks out the suspicious waves for further processing so as to greatly reduce the data to be analyzed further. Nonlinear technique combined with adaptive prediction is developed for this purpose. Here, we use 10-s-long windows to segment the continuous raw EEG. 2) Analytical stage employs multiple methods to detect and classify the epileptic waves from the suspicious segments selected by the first stage. Segments with different

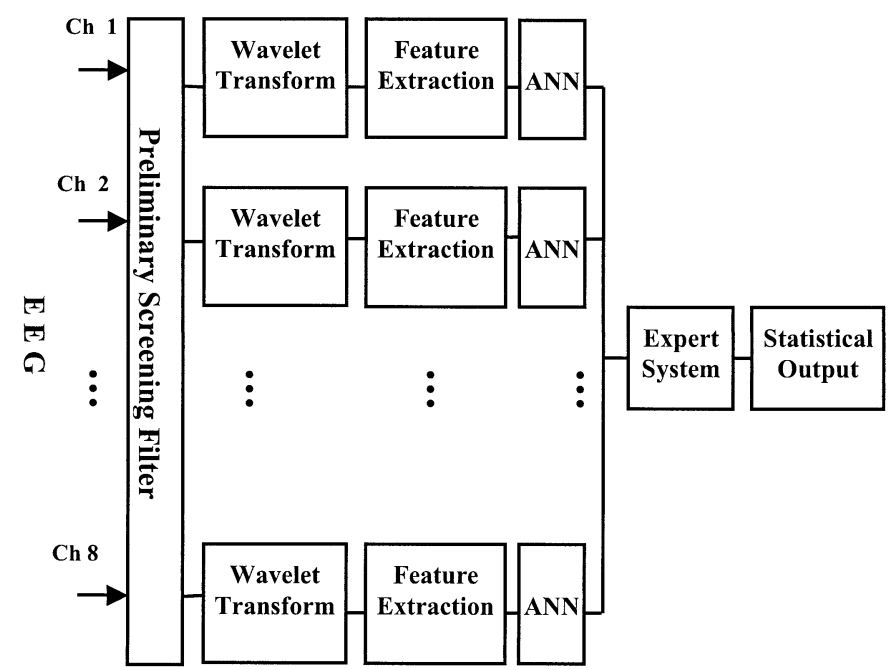

Fig. 1. Block diagram of the automatic detecting system.

length picked out from the first stage are individually analyzed by wavelet transform and artificial neural network, then all these individual segments in the range of 10-s-long window are subjected to expert system to form complexes, or to reject artifacts, etc.

This paper consists of seven sections. The preliminary screening procedure is described in Section II. Section III introduces the wavelet transform we employed. Section IV introduces the structure and learning algorithm of the ANN we used. Section V describes the details of the expert system. Results are presented in Section VI. Section VII contains some discussion.

\section{PRELIMINARY SCREENING}

Epileptic EEG is considered to be mixture of stationary waves and nonstationary transients; they can be separated by the following filtering scheme. The original structure of this filter is proposed in [27]. We improved it to fit the need for the separation of different components of the epileptic EEG (Fig. 2).

As shown in Fig. 2, let EEG time series $x_{n}$ pass through the filter, the signal will be separated into two parts. The nonstationary output $\hat{z}_{n}$ contains spikes, sharp waves, artifacts and some high amplitude slow waves. The stationary part $\hat{y}_{n}$ contains the normal EEG background activities and relatively lowamplitude slow waves.

In this scheme, the key component is the adaptive predictor. It uses the stationary output $\hat{y}_{n}$ to estimate the next input $x_{n}$. The estimation error is $e_{n}$. Suppose the input becomes nonstationary, then $\left|e_{n}\right|$ will go up. In Fig. 2, the stationary output is given by

$$
\hat{y}_{n}=\tilde{y}_{n}+F\left(e_{n}\right)
$$

and the nonstationary output is given by

$$
\hat{z}_{n}=e_{n}-F\left(e_{n}\right)
$$

where $F(\bullet)$ is a nonlinear function (Fig. 3).

When estimation error $e_{n}$ is small $\left(\left|e_{n}\right| \leq \varepsilon_{1}\right)$, it can be inferred from Fig. 3 that $F\left(e_{n}\right)=e_{n}$, so the nonstationary output 


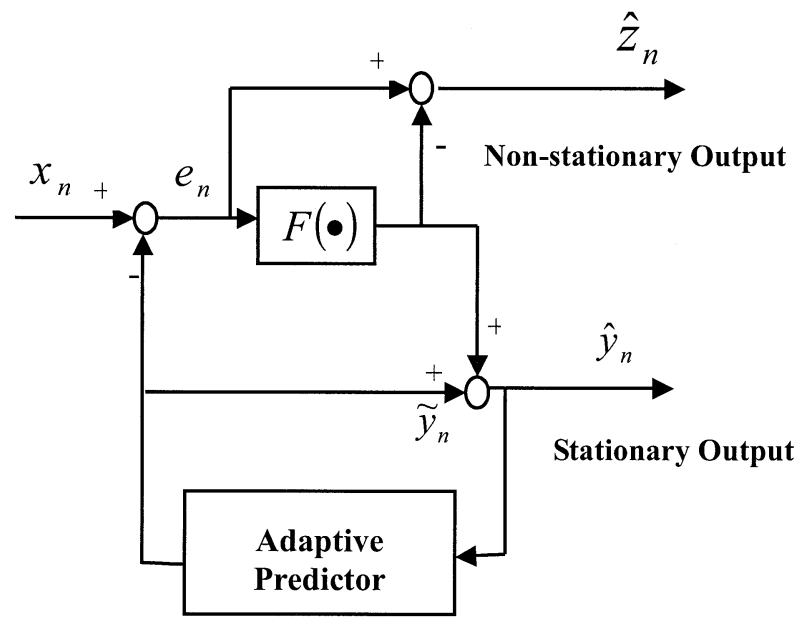

Fig. 2. Filter separates the stationary and nonstationary components of EEG signal. Different types of epileptic waves will appear in these two sections, respectively.

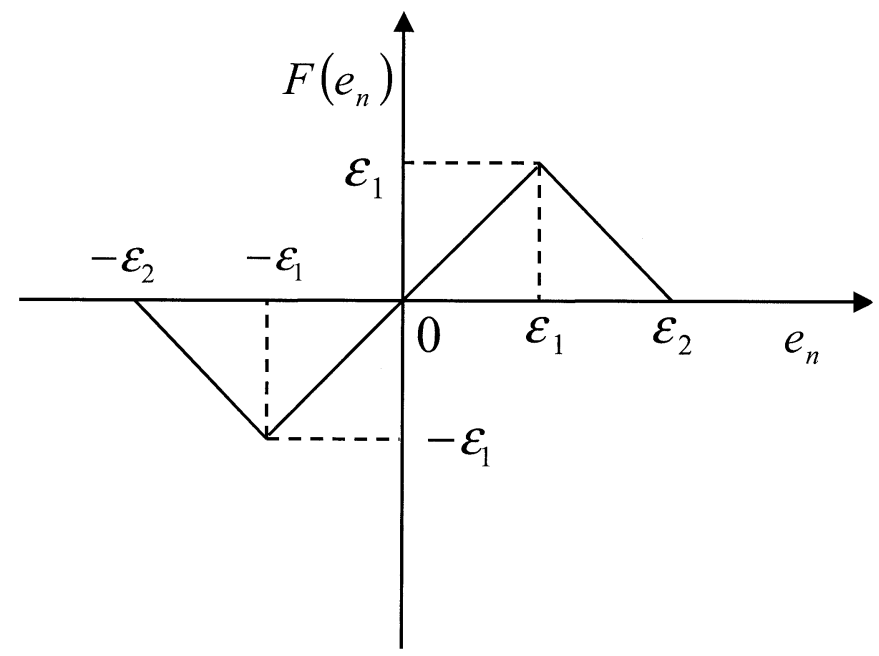

Fig. 3. Nonlinear function $F(\bullet)$ used in preliminary screening. $e_{n}$ means the estimation error.

$\hat{z}_{n}$ will become zero and the stationary output $\hat{y}_{n}=\tilde{y}_{n}+e_{n}=$ $x_{n}$. In this case, the input signal is considered to be stationary.

When $e_{n}$ is large enough $\left(\left|e_{n}\right| \geq \varepsilon_{2}\right), F\left(e_{n}\right)$ will become zero, therefore, the nonstationary output $\hat{z}_{n}=e_{n}$. And the stationary output $\hat{y}_{n}=\tilde{y}_{n}$ (the predicted stationary component).

The effectiveness of the separation depends on the structure and parameters of the adaptive predictor in Fig. 2. In this paper, we propose a predictor based on ANN technique (Fig. 4).

This predictor is a three-layered feed-forward perceptron. Estimation at time $n$ is given by

$$
\tilde{y}_{n}=\sum_{i=1}^{m} d_{n}^{i} \cdot w_{n}^{i(2)}
$$

where $w$ represents the weight coefficient, $m$ is the number of neurons in the hidden layer (Fig. 4), and $d_{n}^{i}$ is the output of the $i$ th neuron in the hidden layer

$$
d_{n}^{i}=f\left[\sum_{j=-k}^{k} \hat{y}_{n+j} \cdot w_{n}^{i, j(1)}\right]
$$

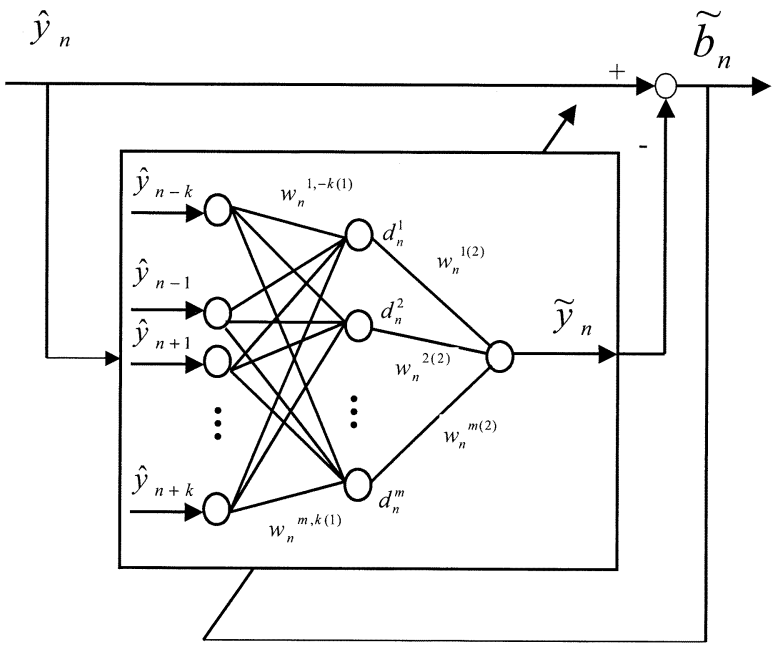

Fig. 4. Adaptive predictor based on ANN; the parameters will be adapted by $\mathrm{BP}$ algorithm.

where

$$
\begin{array}{ll}
j & \neq 0 ; \\
i & =1, \ldots, m ; \\
f(\bullet) & \text { sigmoid function: } f(x)=1 /\left(1+e^{-x}\right) .
\end{array}
$$

In fact, this predictor is a noncausal filter, which estimates the signal at time $n$ using the signals around this time point. Estimation is more accurate using noncausal filter than using causal filter; the noncausal technique is feasible because our system does the analysis offline.

The weight coefficients of this multilayered perceptron are dynamically adjusted according to the estimation error $\tilde{b}_{n}$

$$
\tilde{b}_{n}=\hat{y}_{n}-\tilde{y}_{n} .
$$

The back-propagation (BP) algorithm is the most widely used method for adjusting the weights in ANN. We modify it by employing the delta adaptation (DA) algorithm, which is basically a second-order BP algorithm [16] The DA algorithm can be formulated as follows.

For the output layer

$$
\begin{aligned}
\tilde{\delta}_{n} & =\tilde{y}_{n}\left(1-\tilde{y}_{n}\right)\left(\hat{y}_{n}-\tilde{y}_{n}\right) \\
\Delta A_{n} & =\lambda \Delta A_{n-1}+\gamma \tilde{\delta}_{n}+\sigma \Delta A_{n-2} \\
\Delta w_{n+1}^{i(2)} & =2 \mu \Delta A_{n} d_{n}^{i}+\alpha \Delta w_{n}^{i(2)} .
\end{aligned}
$$

For the hidden layer

$$
\begin{aligned}
\delta_{n}^{i} & =d_{n}^{i}\left(1-d_{n}^{i}\right) w_{n}^{i(2)} \tilde{\delta}_{n} \\
\Delta A_{n}^{i} & =\lambda \Delta A_{n-1}^{i}+\gamma \delta_{n}^{i}+\sigma \Delta A_{n-2}^{i} \\
\Delta w_{n+1}^{i j(1)} & =2 \mu \Delta A_{n}^{i} \hat{y}_{n-j}+\alpha \Delta w_{n}^{i j(1)}
\end{aligned}
$$

where $\Delta A_{n}^{i}$ and $\Delta A_{n}$ are auxiliary variables initialized randomly; $\gamma$ is the learning coefficient, $\lambda$ is the step length, and $\sigma$ and $\alpha$ are the factors for the momentum terms. We set $\gamma=0.8$, $\lambda=0.2, \sigma=0.2$, and $\alpha=0.9$ in our system according to Weng's simulation research [16] $\boldsymbol{\mu}_{n}$ is an adaptive factor that affects the converging speed, and it is calculated as

$$
\boldsymbol{\mu}_{n}=\frac{\boldsymbol{\mu}_{0}}{1+\mu_{0} \cdot \boldsymbol{\eta}_{n}}
$$


where $\boldsymbol{\eta}_{n}$ is approximately the energy of $\hat{y}_{n}$ in the window

$$
\boldsymbol{\eta}_{n}=\sum_{m=-k}^{k} \hat{y}_{n-m}^{2}, \quad m \neq 0 .
$$

$\mu_{0}$ is a constant derived from training. It is the convergence factor when energy $\boldsymbol{\eta}_{n}$ is zero. After optimizing by the "golden section method" [28] with the training data, $\mu_{0}$ is set to 0.00005 .

For the nonstationary component $\hat{z}_{n}$, we set a dynamic threshold of the amplitude based on moving average technique. The threshold of the $n$th point $T h_{n}$ is calculated by

$$
T h_{n}=T h_{n-1} \times \xi+\operatorname{fabs}\left(\hat{z}_{n}-\text { Aver }_{n}\right) \times(1-\xi)
$$

where $\xi$ is the regression factor. We select $\xi=0.9967$ after optimizing with the training data. $f a b s()$ means absolute value; $\operatorname{Aver}_{n}$ is the moving average value of nonstationary output $\hat{z}_{n}$

$$
\operatorname{Aver}_{n}=\operatorname{Aver}_{n-1} \times \xi+\hat{z}_{n} \times(1-\xi) .
$$

It should be noted that the threshold $T h_{n}$ and moving average value Aver $_{n}$ do not refresh when the wave exceeds threshold until the wave falls below the threshold again. The initialization of $T h_{n}$ and $A v e r_{n}$ can both be set to the mean value of a relatively smooth segment in $\hat{z}_{n}$.

If any wave in $\hat{z}_{n}$ exceeds the threshold, the episode will be recorded, and the original EEG data in this episode will then be sent to the analytical stage for further analysis.

For the stationary part $\hat{y}_{n}$, we first smooth it by averaging

$$
g_{n}=\frac{1}{9} \sum_{k=-4}^{+4} \hat{y}_{n-k} \text {. }
$$

Again, a dynamic amplitude threshold is adopted to decide which segment of $g_{n}$ should be recognized as the low amplitude slow wave and sent to the analytical stage. The dynamic threshold is calculated in the same way as that for nonstationary output $\hat{z}_{n}$.

Fig. 5 shows a segment of the original EEG $x_{n}$, with the corresponding nonstationary component $\hat{z}_{n}$, and the stationary component after smoothing, $g_{n}$. It is clear that sharp transients and high amplitude slow waves have been separated into the nonstationary section, whereas the low-amplitude slow waves have been separated into the stationary section. Background EEG is suppressed and slow epileptic waves become more prominent in the signal $g_{n}$ (Fig. 5).

In order to test the efficiency of preliminary screening, eight 1-h-long 16-channel EEG records from eight typical epileptic patients were processed. The results were compared with the report made by human experts. According to this test, all the 1018 sharp transients and 9117 out of the 9398 slow waves were detected by the preliminary screening stage. In this stage, the detection rate of slow wave was $97.0 \%$, and the detection rate of all the epileptic waves was $97.3 \%$, whereas the data volume was reduced to about $25 \%$.

\section{WAVELET TRANSFORM}

Wavelet transform is a multiresolution analysis tool that can disclose the characteristics of the signal in joint time-frequency domain. It is quite suitable for processing of nonstationary

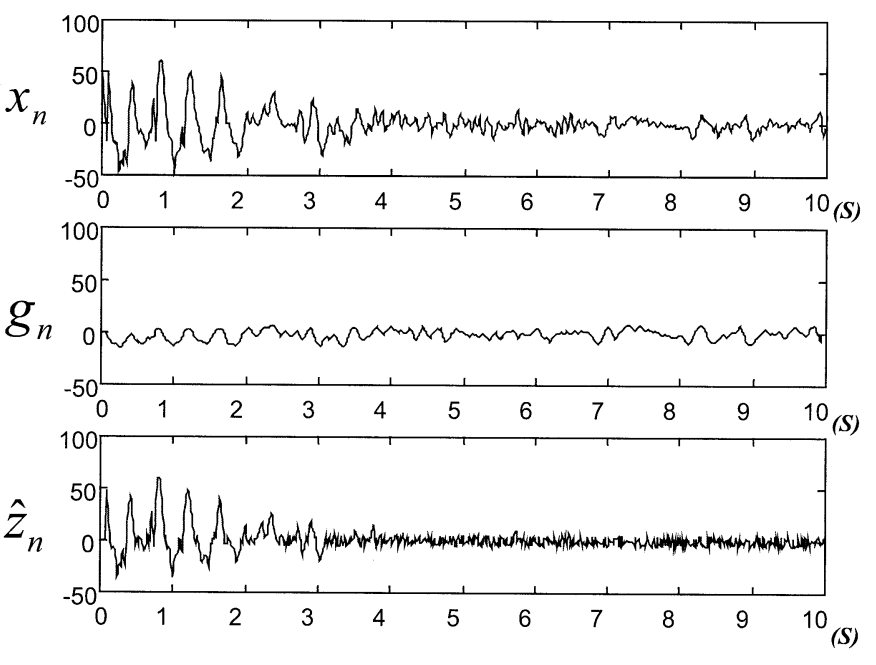

Fig. 5. Result of stationary and nonstationary components separation. $x_{n}$ is the raw EEG, $\hat{z}_{n}$ is the nonstationary component, $g_{n}$ is the stationary component after smoothing. Nonstationary component consists of sharp transients and high amplitude slow waves. Stationary component consists of relatively low-amplitude slow waves.

signal and has been successfully applied to the analysis of EEG [19]-[21] and other biological signals [29].

Discrete wavelet transform (DWT) is widely used because it demands relatively less computation compared to continuous wavelet transform (CWT). The main limitation of DWT is the roughness of its scale increment which doubles itself between two adjacent levels. However, CWT can achieve finer scale sampling so it is able to analyze signal with higher frequency resolution and that is why we adopt it in our EEG system.

CWT of time series $x(t)$ is defined as

$$
\operatorname{CWT}\{x(t) ; a, b\}=\frac{1}{\sqrt{a}} \int_{-\infty}^{\infty} x(t) \psi^{*}\left(\frac{t-b}{a}\right) d t
$$

where $\psi(t)$ denotes the mother wavelet, " $*$ " indicates complex conjugate, $a$ represents the chosen wavelet scale, $b$ is the timeshifting parameter.

By varying the wavelet scale $a$, wavelet transform decomposes the signal $x(t)$ into different frequency bands according to the value of $a$; larger $a$ corresponds to lower frequency but broader field of view, whereas smaller $a$ corresponds to higher frequency but narrower field of view.

The computation load of calculating CWT directly from its definition (17) is quite demanding. So fast algorithm of it is necessary. Some fast CWT algorithms have been developed, such as that based on chirped $\mathrm{Z}$ transform [30], and that based on filter bank [31].

We have proposed a fast CWT algorithm based on the Mellin transform, which calculates wavelet coefficients of different scales simultaneously at a given time instant [32], whereas other algorithms calculate wavelet coefficients of different time instants consecutively at a given scale [30], [31], [33]. Since the data have been segmented into different length after preliminary screening stage, it is more appropriate to compute wavelet transform by the first approach. Only two inverse FFTs and one FFT are needed for each calculation. A detailed discussion about this algorithm can be found in [32]. 


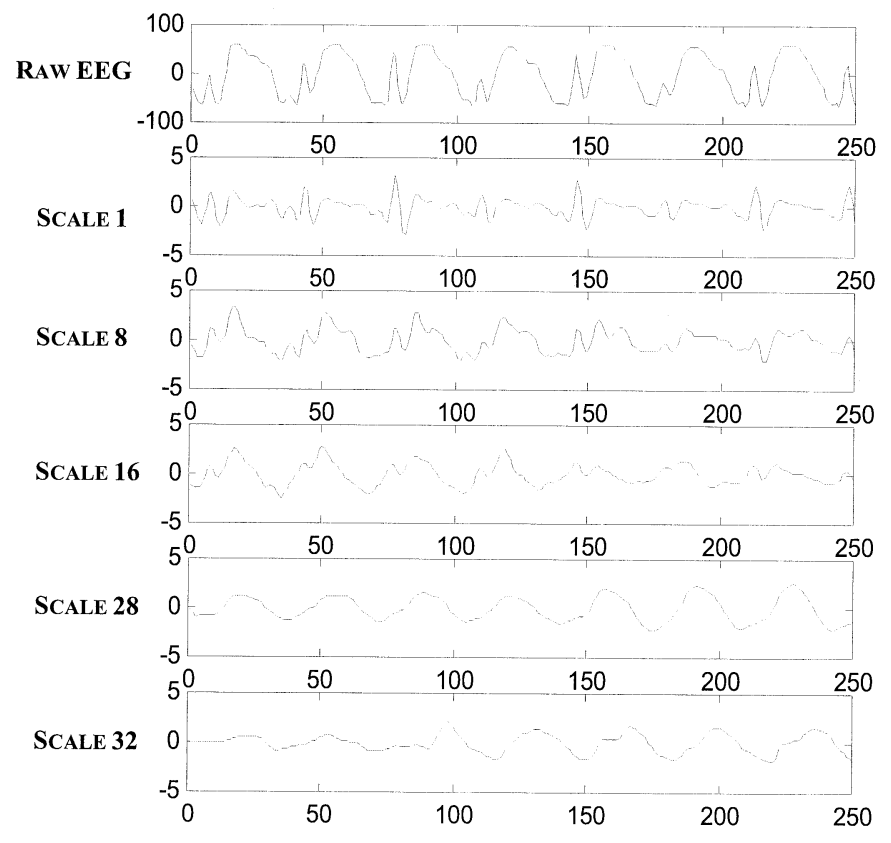

Fig. 6. EEG signal is decomposed by wavelet transform. High-frequency components appear in smaller scales, low-frequency components appear in larger scales.

Thirty-two scales are used to decompose the EEG segments selected by the preliminary screening stage. The scale parameter $a$ is sampled geometrically as

$$
a=q^{n}, \quad n=1 \sim 31
$$

where $q$ is the ratio between adjacent levels. It is set to 1.06 in our system, thus, the scales span from $a=1$ to $a=6.45$, which are wide enough to cover all the useful frequency components in EEG.

Marr wavelet is selected as the mother wavelet, which is defined as

$$
\psi(t)=\left(1-t^{2}\right) e^{-t^{2} / 2}
$$

Fig. 6 shows the result of wavelet transform. The topmost curve is an original record of EEG with epileptiform discharge. It is decomposed into three basic components (spikes, sharp waves, and slow waves) by CWT. The curves below the topmost one are wavelet coefficients in different scales. It can be clearly seen that as the scales increase, the frequency components of EEG are resolved in an orderly way-from high frequencies to low frequencies.

After wavelet transform, spikes are prominent in the eight lowest scales (scale index 0-7), sharp waves appear in the eight medium scales (scale index 4-11), and slow waves appear in the four higher scales (scale index 24-27). Different thresholds are set for these scales. If any wave exceeds the threshold, it will be picked out for further processing.

We use background amplitude of wavelet coefficients as the threshold. In the $n$th scale the background is denoted as $w_{T}^{n}(t)$.
At every time point $t$, its value is dynamically adjusted in a similar way as equations (14) and (15)

$w_{T}^{n}(t)=w_{T}^{n}(t-1) \times \xi+f a b s\left(w^{n}(t)-\operatorname{Aver}^{n}(t)\right) \times(1-\xi)$

where $w^{n}(t)$ is the wavelet coefficients, $\operatorname{Aver}^{n}(t)$ is the moving average value of wavelet coefficients in scale level $n$

$$
\operatorname{Aver}^{n}(t)=\operatorname{Aver}^{n}(t-1) \times \xi+w^{n}(t) \times(1-\xi) .
$$

The regression factor $\xi$ is set to 0.9933 based on our training data.

The background amplitude $w_{T}^{n}(t)$ and moving average value $\operatorname{Aver}^{n}(t)$ do not refresh when the wave exceeds background until the wave falls below the background amplitude again. The initialization of $w_{T}^{n}(t)$ and $\operatorname{Aver}^{n}(t)$ can both be set to the mean value of a relatively smooth segment in $w^{n}(t)$.

Wavelet transform is also an effective way to reject artifacts. After the transform, many artifacts will only appear in one or two scales, but they will not appear in as many adjacent scales as epileptic waves do. Therefore, the artifacts can be rejected from the signal if multiscale information is employed.

From above processing, the three basic components of epileptic waves have been decomposed into three different groups of scales; hence the subsequent processes can be designed individually for each group.

\section{Feature Extraction AND ARtificial NEURAL NETWORK}

\section{A. Feature Extraction}

If the wavelet coefficients of the predefined scales are to be analyzed further, some feature parameters of the wavelet coefficients must be extracted first. These parameters will then act as the input of the ANN. ANN will decide whether the suspicious wave is similar to epileptic wave and how great the similarity is.

We use waveform features of wavelet coefficients rather than wavelet coefficients per se or raw EEG data directly as the input of ANN. Because EEG waveforms are quite different from patient to patient, if we use the wavelet coefficients or raw EEG data as input, it is almost impossible for the network to cluster these various waveforms. Ko et al. [17] have concluded that spike detection using raw EEG data as input of ANN is unlikely to be feasible under current computer technology.

Feature parameters are defined as the following (Fig. 7).

1) Relative amplitude $1\left(R A_{1 u}\right.$ and $\left.R A_{1 d}\right)$ : Voltage difference between the peak and valleys of a wave divided by the background amplitude (the subscript $u$ stands for the ascending branch and the subscript $d$ for the descending branch)

$$
R A_{1 u}=\left|a_{C A}\right| / w_{T}^{n}(p), \quad R A_{1 d}=\left|a_{C B}\right| / w_{T}^{n}(p)
$$

where $w_{T}^{n}(p)$ is the background amplitude of wavelet coefficients at the time when the wave reaches the peak. The background amplitude is calculated using (20) and (21).

2) Relative amplitude $2\left(R A_{2 u}\right.$ and $\left.R A_{2 d}\right)$ : The potential difference between the peak and turning points divided by background amplitude

$$
R A_{2 u}=\left|a_{C D}\right| / w_{T}^{n}(p), \quad R A_{2 d}=\left|a_{C E}\right| / w_{T}^{n}(p) .
$$




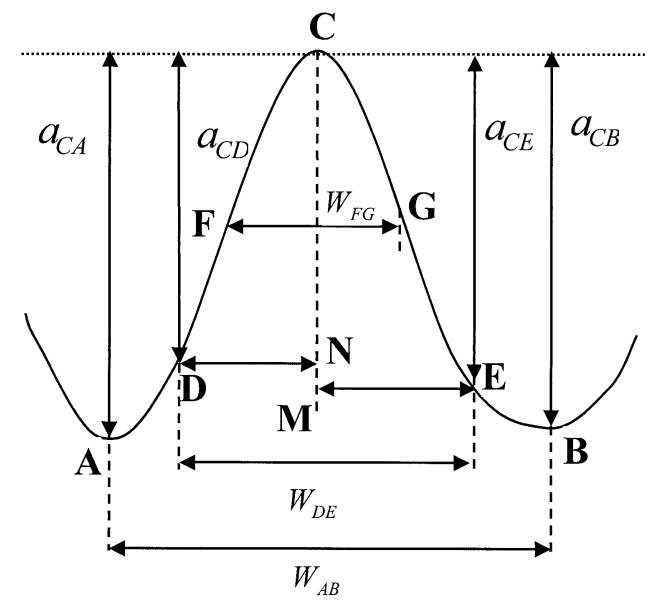

Fig. 7. Feature parameters as the input of ANN. Different sets of parameters are used for different types of wave.

Turning point is defined as the point where the slope decreases more than $50 \%$ compared to that of the preceding point for the first time (starting from the peak point).

3) Duration $1\left(W_{1}\right)$ : Number of data points between the start and the end points of the wave, shown as $W_{A B}$ in Fig. 7.

4) Duration $2\left(W_{2}\right)$ : Number of data points between the two turning points of the wave, shown as $W_{D E}$ in Fig. 7.

5) Duration $3\left(W_{3}\right)$ : Distance between the two "half-maximum points." Half-maximum points are points where the amplitudes are half of $\left|a_{C A}\right|$ or $\left|a_{C B}\right|$. Refer to $W_{F G}$ in Fig. 7.

6) Sharpness $(\mathrm{SH})$ : Sharpness is the changing rate of the slope at the peak point. If the peak point is denoted as $k$, $S H$ can be calculated as

$$
S H=[s l(k+1)-s l(k-1)] / 2
$$

where $\operatorname{sl}(k)$ is the slope at point $k$

$$
s l(k)=\left[w^{n}(k+1)-w^{n}(k-1)\right] / 2 .
$$

$w^{n}(k)$ means the $k$ th point of the wavelet coefficients of scale $n$.

7) Slope ( $S L P_{1}$ and $\left.S L P_{2}\right)$ : Slope of the lines connecting the peak and the two turning points (refer to Fig. 7)

$$
S L P_{1}=C N / D N, \quad S L P_{2}=C M / E M .
$$

For the three basic epileptic components, we use different set of parameters. For spike and sharp wave, seven parameters $\left(R A_{2 u}, R A_{2 d}, S L P_{1}, S L P_{2}, W_{1}, W_{2}, S H\right)$ are used; for slow wave, four parameters $\left(W_{1}, W_{3}, R A_{1 u}, R A_{1 d}\right)$ are used.

\section{B. Artificial Neural Network and "Twice-Learning” Algorithm}

Three similar MLP neural networks are designed respectively for detection of spikes, sharp and slow waves. Each has different number of input nodes, hidden layer nodes and one output node. The inputs of the network are those feature parameters acquired above and the output indicates the proximity between the input

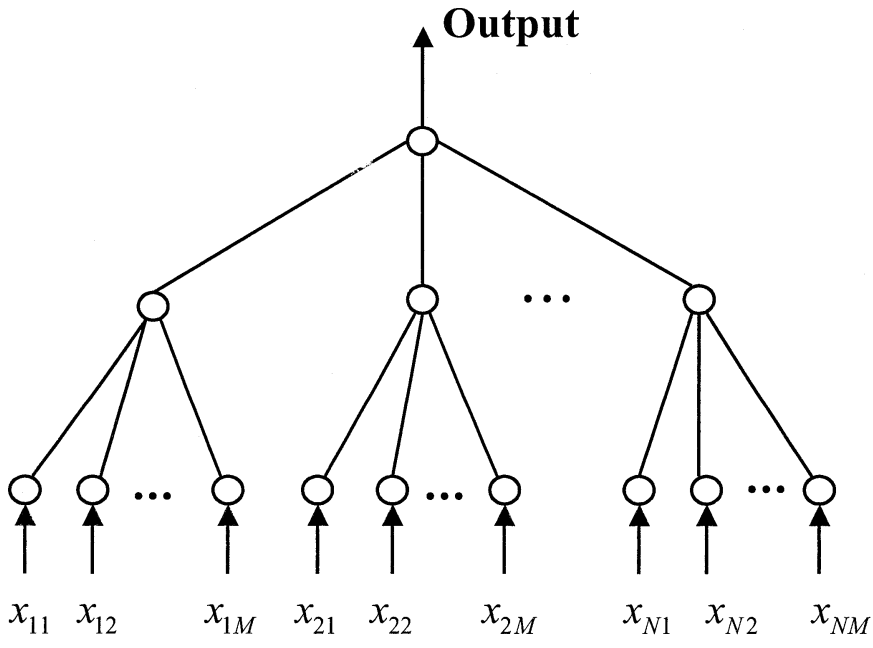

\section{$\begin{array}{lll}\text { Scale } 1 & \text { Scale } 2 & \text { Scale N }\end{array}$}

Fig. 8. Structure of ANN. Number of input nodes depends on the type of epileptic wave. For spike, number of input nodes is $7 \times 8=56$; for sharp wave, it is $7 \times 8=56$; and for slow wave, it is $4 \times 4=16$.

wave and the corresponding basic epileptiform waves. In practice, it is more reliable to give the likelihood of a wave being epileptic than declaring yes-or-no categorically. Hence, in our system linear output is used to take the place of binary output, so that the value of the output gives the proximity of the input wave.

In order to gain linear output from the network, some scholars set the desired output by statistical distance of the training data [34]. The training process is not reliable because the desired output does not reflect the pattern of input objectively. This paper proposes an efficient "twice-learning" algorithm to achieve linear output from the three-layer BP neural network and to reduce human interference.

The structure of the ANN is illustrated in Fig. 8. $N$ is the number of scales we use; it is also the number of hidden layer nodes. (i.e., for spikes: $N=8$, sharp waves: $N=8$, slow waves: $N=4$ ). $M$ is the number of input parameter (i.e., for spikes: $M=7$, sharp waves: $M=7$, slow waves: $M=4$ ). $X_{i j}$ is the $j$ th parameter extracted from the $i$ th scale. The order of the parameters is the same for all scales.

The "twice-learning" algorithm is described as following:

In the first step, the desired outputs are binary and set by human expert. The networks update their coefficients using weighted BP learning algorithm. When the training is finished, the networks have the ability to separate the data roughly into two groups. The outputs will be very close to one or zero. The closer a wave approximates the pattern of an epileptic wave, the larger will be the output, and vice versa.

In the second step, the outputs of all the "positive" samples are sorted, then the maximum and minimum output, denoted as $P_{\max }$ and $P_{\min }$, are found. For a "positive" sample with output $y$, its desired output for the second round training is defined as

$$
P O_{y}=\frac{y-P_{\min }}{P_{\max }-P_{\min }} \times 0.45+0.55 .
$$

Thus, all the "positive" samples will have desired output falling in the range 0.55 to 1 . 
Similarly, we find the maximum and minimum output of "negative" samples, denoted as $N_{\max }$ and $N_{\min }$, then define the desired output as

$$
N O_{y}=\frac{y-N_{\min }}{N_{\max }-N_{\min }} \times 0.45 .
$$

The network is then trained again using the new desired output; the coefficients are also updated by weighted BP learning algorithm.

The "twice-learning" algorithm proved to be efficient for training. The first step provides objective output for the second step, and increases the convergence speed as well. The second step enables the network to produce linear output according to the pattern of input data. It successfully avoids the artificial interference in training, thus maintaining the objectivity and congruity of the output.

After the two steps, the network can give the proximity of a wave to a specific epileptiform wave: spike, sharp or slow wave. All waves having proximity higher than 0.35 are sent to expert system for further analysis. We choose such a low threshold mainly for two reasons: 1) epileptic waves often appear in multiple channels; in some channels the waves have high amplitudes but in some other channels they may have low amplitudes, thus, their proximity might be small; and 2) some parts in complex waves are not prominent, e.g., spike may be covered by slow wave or slow wave can be very small in complex waves. These waves will not be missed by a low threshold strategy and expert system will then enhance their proximity according to the heuristic rules.

\section{EXPERT SYSTEM}

Expert system fully exploits the spatial and temporal contextual information in order to reject artifacts. Several heuristic rules are combined to distinguish artifacts (such as eye blink, electrode movement or other artifacts) from epileptic waves. Thereafter, some isolated waves are reconstructed into spike-and-slow complex or sharp-and-slow complex. Finally the decision is made as to whether the wave is a kind of epileptic wave and, if so, to which kind it belongs. The rules we have used are listed as follows.

1) Adjacent spike and slow wave or adjacent sharp and slow wave should be combined into complex waves.

2) Slow waves in Fp1 and Fp2 are more likely to be eye blinks.

3) Epileptic waves usually do not achieve their peaks exactly at the same time in all channels.

4) Epileptic waves usually do not appear solely, neither spatially nor temporally.

5) $\alpha$ wave is easy to be confused with sharp waves, but it mainly occurs in $\mathrm{O} 1$ and $\mathrm{O} 2$, and may appear to be consecutive sharp waves.

6) $K$-complex during sleep is a kind of slow wave but usually overridden by spindles (appear to be consecutive small sharp waves here).

7) Normal slow waves in sleep stages should not be falsely judged.
More heuristic rules can be easily added into our system so it is possible to mimic the judging procedure of human expert better in the future. The above rules are implemented in following three aspects.

\section{A. Integration of Multiscale Information}

Components of different frequency have been separated into different scales after wavelet transform. This step integrates the waves of these scales. All of the waves whose output from neural network exceeds the threshold 0.35 are put into a queue; some adjacent waves are then combined into complex waves (e.g., spike-and-slow complex or sharp-and-slow complex); some overlapped waves are classified accordingly.

\section{B. Spatial Information Integration}

Eye blinks can be distinguished in the frontal channels. If some high-amplitude slow waves are found simultaneously in channels $\mathrm{Fp} 1$ and $\mathrm{Fp} 2$, and their durations are longer than $200 \mathrm{~ms}$, they will be rejected as eye blinks.

Epileptic waves in different channels always have similar patterns, but their peaks usually do not appear exactly at the same time, i.e., a time delay could be found between the two peaks. Thus, we could discriminate between transients caused by electrode movement and real epileptic transients based on this fact. A sudden movement of electrode may cause a waveform similar to sharp wave in many channels, but their peaks usually appear in all channels at the same time point. If this case occurs, and around this transient there is no other epileptic waves, then it should be rejected as artifacts caused by electrode movement.

If a wave has similar patterns in many channels (e.g., their starts, ends, and peaks are very close) then its proximity should be increased by 0.2 . Conversely, if a wave has no "image" in other channels, its proximity will be decreased by 0.2 . The increase or decrease of the proximity is not arbitrarily set but decided by training. It is chosen to be 0.2 on our training data.

Some $\alpha$ waves overlap with sharp waves in frequency domain so they may cause confusion. Fortunately, $\alpha$ waves mostly appear in channels $\mathrm{O} 1$ and $\mathrm{O} 2$, hence, when more than three adjacent sharp waves appear in these two channels, and their proximity are not very large, then they should be rejected as $\alpha$ waves.

\section{Use of Temporal Context}

Proximity of a wave will be increased if it has temporal support for its existence, otherwise the proximity will be decreased.

If a spike has rather small proximity, but it is followed by a slow wave with large proximity, then the proximity of the spike should be increased by 0.2 .

If a sharp wave or spike appears solely and there is no other epileptiform waves in the previous or following $1 \mathrm{~s}$, then proximity of this wave should be decreased by 0.2 .

If a slow wave in frontocentral area has high-amplitude longduration biphasic waveform with overriding consecutive small sharp waves, we will take this slow wave as $K$-complex in sleep stage and eliminate it from epileptic waves.

During the sleep hours according to patient's diary, the threshold for slow waves will be set to 0.95 . This is because slow waves can be normal waveforms in sleep stages. Thus, 
only a small number of slow waves with very high likelihood during sleep hours will be picked out for human review.

Every suspicious wave should be tested again according to the definition of spike, sharp wave and slow wave. Only those agree with the definition in duration, amplitude, and symmetry will be retained.

\section{RESULTS}

The system was evaluated using 8-channel clinical EEG records of 81 epileptic subjects. The data were chosen from the patient databases of four hospitals; none of them had been used previously in system development, and the data were not pre-selected in any way. Thus, the testing procedure can reflect the real performance of our system in clinical application.

The records ranged from 4 to $24 \mathrm{~h}$ in length, totaling more than $800 \mathrm{~h}$. The data contained all types of epileptiform activities, including focal and nonfocal epileptic EEG, e.g., single spikes, spike bursts, spike-slow wave bursts, sharp waves, slow wave bursts, and sharp-slow waves, all in various amplitudes. Almost all kinds of artifacts that commonly appear in AEEG can be found in our data, such as artifacts caused by eye blink, muscle strengthen, electrode movement, etc. The placement of the electrodes was identical for every record. But the records differed in length and were gathered by different EEG machines, so we had converted the data into same format. The sampling rate was $100 \mathrm{~Hz}$ and $\mathrm{A} / \mathrm{D}$ conversion precision was 8 bit.

For comparison with analysis of human experts, the data were subjected to computer analysis and human review independently. Reports made by human experts were taken as the reference. In the computer analysis, all events having proximity larger than a threshold were reported and compared with the result of human review. The threshold was set to 0.80 to achieve best compromise between sensitivity and specificity on our training data (except the slow waves during sleep stages, for these waves the threshold was 0.95). It might change slightly for other training data. We also provided a user interface in our system which enables the user to multiply a factor $\psi$ to the threshold, thus the final threshold will be $0.8 \times \psi$. By adjusting the factor $\psi$ the EEGer can manually change the threshold to achieve better result on a specific subject when it is needed.

We divided these 81 records into three groups.

Group 1) Records dominated by slow waves.

Group 2) Records dominated by spikes, sharp waves, and complexes.

Group 3) Records contaminated by many artifacts.

Table I illustrates the result of the analysis.

In Group 1, spikes and sharp waves rarely appeared. Slow waves became the most important indication of events. As slow waves have been fully considered in our system, the epileptiform activities can be correctly pointed out. Records in this group amounted to $450 \mathrm{~h}$ in length, in which human experts found 86434 events. $88.9 \%$ of these events were detected by ours system and the false detection rate was $5.6 \%$. The false detection rate was calculated as

False Detection Rate $=\frac{\text { False Positive Events }}{(\text { All Events Found By Human Experts })}$
TABLE I

Statistics OF System PeRformanCE

\begin{tabular}{|c|c|c|c|c|c|c|c|c|c|}
\hline \multirow{2}{*}{$\begin{array}{c}\text { EEG } \\
\text { Group } \\
\text { No. }\end{array}$} & \multicolumn{2}{|c|}{$\begin{array}{l}\text { No. of Isolated } \\
\text { Spikes and } \\
\text { Sharp Waves }\end{array}$} & \multicolumn{2}{|c|}{$\begin{array}{l}\text { No. of Spike-slow } \\
\text { Complex Waves } \\
\text { and Sharp-slow } \\
\text { Complex Waves }\end{array}$} & \multicolumn{2}{|c|}{$\begin{array}{c}\text { No. of } \\
\text { Slow Waves }\end{array}$} & \multirow[t]{2}{*}{$\begin{array}{l}\text { No. of } \\
\text { False } \\
\text { Detected } \\
\text { Waves }\end{array}$} & \multicolumn{2}{|c|}{$\begin{array}{c}\text { All Types of } \\
\text { Epileptic Waves }\end{array}$} \\
\hline & Total & $\begin{array}{l}\text { Correctly } \\
\text { Detected }\end{array}$ & Total & $\begin{array}{l}\text { Correctly } \\
\text { Detected }\end{array}$ & Total & $\begin{array}{l}\text { Correctly } \\
\text { Detected }\end{array}$ & & $\begin{array}{l}\text { False } \\
\text { Rate }\end{array}$ & $\begin{array}{c}\text { Detection } \\
\text { Rate }\end{array}$ \\
\hline 1 & 1756 & 1665 & 3349 & 3304 & 81329 & 71886 & 4801 & $5.6 \%$ & $88.9 \%$ \\
\hline 2 & 3940 & 3800 & 31683 & 31114 & 13218 & 10201 & 3112 & $6.4 \%$ & $92.4 \%$ \\
\hline 3 & 352 & 337 & 1330 & 1319 & 8273 & 7122 & 1005 & $10.1 \%$ & $88.2 \%$ \\
\hline $\begin{array}{l}\text { Sunmation } \\
\text { of All } \\
\text { Grouns }\end{array}$ & 6048 & 5802 & 36362 & 35737 & 102820 & 89209 & 8918 & $6.1 \%$ & $90.0 \%$ \\
\hline
\end{tabular}

The result show our system can distinguish most events even the sharp transients are few in EEG.

Group 2 contained many spikes, sharp waves, and complexes, and also large amount of slow waves. Records in this group added up to $350 \mathrm{~h}$ in length, including 48841 events. $98.0 \%$ of the sharp transients were detected correctly. Detection rate of all epileptic events was as high as $92.4 \%$ with a false detection rate of $6.4 \%$. Though slow waves have been paid much attention, sharp transients have not been neglected at all. The results show our system is very sensitive to sharp transients.

Group 3 contained many artifacts that usually hamper the automatic detecting system. However, in our system, most of these artifacts can be recognized and rejected. Records in this group amounted to $60 \mathrm{~h}$ in length. The detection rate in this group reached $88.2 \%$ and the false rate was $10.1 \%$. It can be inferred that the system rejects artifacts satisfactorily.

The overall detection rate of the spikes, sharp waves and complexes was $98.0 \%$ when the results of the three groups were combined. It also proved satisfactory for slow wave recognition even though they are harder to be detected compared to sharp transients; the detection rate of slow wave was $86.8 \%$. Overall, $90.0 \%$ of the epileptic waves were correctly discriminated and classified, whereas the false rate was kept at $6.1 \%$.

We plot the receiver operating characteristic (ROC) curve to evaluate the system performance on the whole testing data set. In our system, the final threshold for detection of epileptic waves is $0.8 \times \psi$. We vary the factor $\psi$ step by step in the range [0.35, 1.25 ] and calculate the detection rate and false rate in every step. Then, we obtain the ROC curve as shown in Fig. 9. The area under ROC curve is about $95 \%$. The area under ROC curve reflects the tradeoff between sensitivity and specificity; a larger area indicates better discrimination ability of the system [35]. The large area under our ROC curve has proved the system can have high sensitivity and high specificity at the same time.

The proposed system also has an attractive processing speed. In our test, the average speed is about 60 times faster than recording speed on a general purpose PIII400 Pentium PC. Thus, a 24-h-long record can be processed in $24 \mathrm{~min}$. It should 


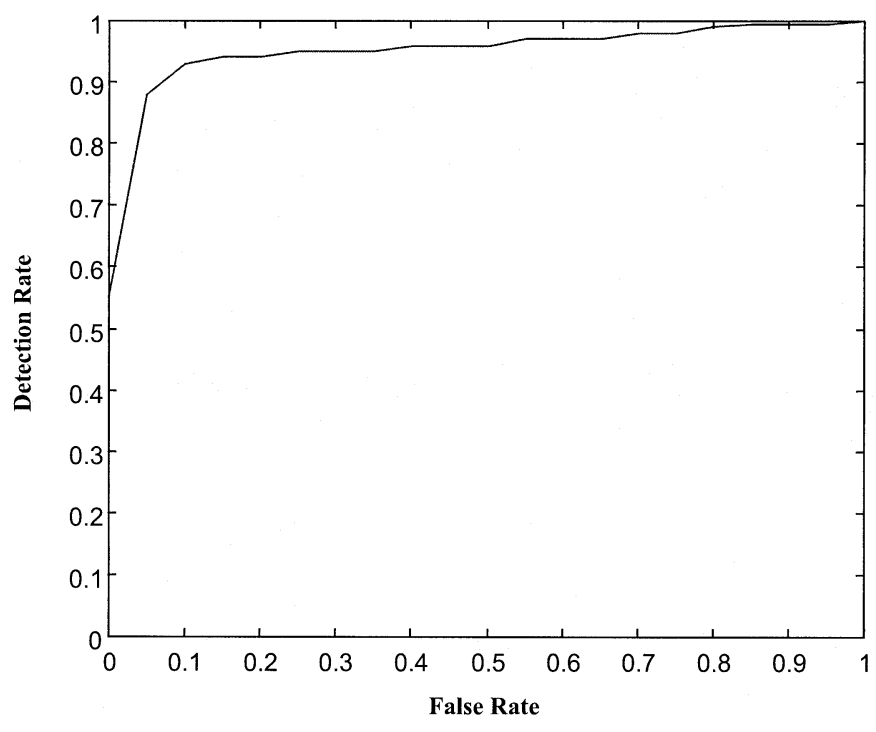

Fig. 9. ROC curve for final threshold. The area under ROC curve is around 0.95 . The large area under the ROC curve indicates the system can have high sensitivity and high specificity at the same time.

be noted the speed also depends on the proportion of epileptic waves in the raw EEG.

\section{DISCUSSION}

In this paper, we set up a scheme for automatic detection of epileptic EEG. Multiple up-to-date signal-processing methods are integrated into one system so that they can complement mutually to improve the overall performance of the system. The system also makes good use of spatial and temporal contextual information while taking care of all kinds of epileptiform waves, including spike, sharp wave, slow wave, and wave complex. The output of the system indicates the type of the wave and the likelihood of the wave to be epileptic.

In order to fit the requirements for clinical use, an automatic detection system should pay more attention to slow epileptiform activities while identifying the sharp transients. The multistage multimethod approach proposed here is quite effective in identifying different kinds of epileptic waves. Even with clear definitions of the scope of amplitude and width, it is not realistic to classify epileptic waves by definitions alone. A good system should take into account the background amplitude and rhythm besides analyzing the morphologies of waves. In our work, we set up dynamic thresholds based on the background activities. However, much work can still be done in the future; for example, the basic rhythm of EEG background in different physiological stages must be considered. A $\delta$ wave may indicate abnormality if it appears on an adult's EEG, but it can be the main rhythm of a healthy child's EEG. Some other physiological or pathological conditions of the subject can also affect the judgment.

Another hard problem is how to avoid misinterpretation of waves during sleep. Beun et al. [36] found that paroxysmal sharp transients may be recorded during drowsiness or sleep in healthy subjects. During sleep stage I, there will be some positive occipital sharp transients (POSTs) and vertex sharp transients, these are normal sleep patterns and should not be mis- taken for epileptic waves. Moreover, in sleep stage I, stage II, and also in REM sleep, $\theta$ wave will frequently appear in EEG; in sleep stage III and IV, $\delta$ wave will appear with high amplitude. These waves can hardly be discriminated from epileptic slow waves because of their similar morphologies and distributions. Thus, a better system should analyze the sleep stages and adjust the judge standard according to different sleep status. However, automatic sleep staging is also a difficult problem and there is still a long way to go before it can be solved. Currently, we have started the effort to integrate an automatic sleep staging system with the epileptic EEG detection system to obtain a more reliable result.

Maintaining high flexibility of the system is also an important point. Waveforms may differ greatly between patients or between different periods of the same patient, so the system must be flexible in order to retain efficiency under various circumstances. In the present system, we use adaptive prediction in the preliminary screening stage and set self-adaptive thresholds at various points of the analytical stage. The system adjusts its parameters dynamically when the characteristics of the input data change. Thus it is more robust for long-term data processing and for data from different subjects.

"Begin in roughness, end in fineness" is an important philosophy in our design. An efficient strategy in our system is reducing data before the detailed analysis. According to our statistics, the preliminary screening reduces about $75 \%$ of the data; this guarantees the system a fast processing speed. When training the ANN, the "twice-learning" algorithm also validates the philosophy. In the first step, the training dataset are roughly divided into two main groups, in the second step, a linear output is given to indicate the likelihood of a wave to be epileptic. This methodology guarantees the fast convergence of network while achieving linear output.

Based upon these ideas, improvement of our system is just under way.

\section{REFERENCES}

[1] E. J. Waterhouse, "Ambulatory electroencephalography (EEG)," eMed. J., vol. 2, no. 2, Feb. 2001.

[2] W. R. S. Webber, B. Litt, R. P. Lesser, R. S. Fisher, and I. Bankman, "Automatic EEG spike detection: What should the computer imitate," Electroenceph. Clin. Neurophysiol., vol. 87, pp. 364-373, 1993.

[3] W. E. Hosteler, H. H. Doller, and R. W. Homan, "Assessment of a computer program to detect epileptiform spikes," Electroenceph. Clin. Neurophysiol., vol. 83, pp. 1-11, 1992.

[4] P. Y. Ktonas, "Automated spike and sharp wave (SWW) detection," in Methods of Analysis of Brain Electrical and Magnetic Signals. Handbook of Electroencephalography and Clinical Neurophysiology, A. S. Gevins and A. Remond, Eds. Amsterdam, The Netherlands: Elsevier, 1987, pp. 211-241.

[5] R. Sankar and J. Nator, "Automatic computer analysis of transients in EEG," Comput. Biol. Med., vol. 22, no. 6, pp. 407-442, 1992.

[6] J. S. Barlow, "EEG transient detection by matched inverse digital filtering," Electroenceph. Clin. Neurophysiol., vol. 48, pp. 246-248, 1980.

[7] J. Gotman and P. Gloor, "Automatic recognition and quantification of interictal epileptic activity in the human scalp EEG," Electroenceph. Clin. Neurophysiol., vol. 41, pp. 513-529, 1976.

[8] A. Dingle, R. D. Jones, G. J. Carroll, and W. R. Fright, "A multistage system to detect epileptiform activity in the EEG," IEEE Trans. Biomed. Eng., vol. 40, pp. 1260-1268, Dec. 1993.

[9] H. Qu and J. Gotman, "Improvement in seizure detection performance by automatic adaptation to the EEG of each patient," Electroenceph. Clin. Neurophysiol., vol. 86, pp. 79-87, 1993. 
[10] J. R. Glover, P. Y. Ktonas, N. Raghavan, J. M. Urunuela, S. S. Velamuri, and E. L. Reilly, "A multichannel signal processor for the detection of epileptogenic sharp transients in the EEG," IEEE Trans. Biomed. Eng., vol. BME-33, pp. 1121-1128, Dec. 1986.

[11] J. R. Glover, D. N. Varmazis, and P. Y. Ktonas, "Continued development of a knowledge-based system to detect epileptogenic sharp transients in the EEG," in Proc. Conf. IEEE Engineering in Medicine and Biology Society, 1990, pp. 1374-1375.

[12] A. A. Dingle, R. D. Jones, G. J. Carroll, W. R. Fright, and I. M. Donaldson, "The use of multichannel information in the detection of epileptiform activity in the EEG," in Proc. Conf. IEEE Engineering in Medicine and Biology Society, 1990, pp. 849-850.

[13] J. R. Glover, N. Raghavan, P. Y. Ktonas, and J. D. Frost, "Contextbased automated detection of epileptogenic sharp transients in the EEG: Elimination of false positives," IEEE Trans. Biomed. Eng., vol. 36, pp. 519-527, May 1989

[14] A. J. Gabor, R. R. Leach, and F. U. Dowla, "Automated seizure detection using a self-organizing neural network," Electroenceph. Clinic. Neurophysiol., vol. 99, pp. 257-266, 1996.

[15] W. R. S. Webber, R. P. Lesser, R. T. Richardson, and K. Wilson, "An approach to seizure detection using an artificial neural network (ANN)," Electroenceph. Clinic. Neurophysiol., vol. 98, pp. 250-272, 1996.

[16] W. Weng and K. Khorasani, "An adaptive structure neural network with application to EEG automatic seizure detection," Neural Networks, vol. 9, no. 7, pp. 1223-1240, 1996.

[17] C. W. Ko and H. W. Chung, "Automatic spike detection via an artificial neural network using raw EEG data: Effects of data preparation and implications in the limitations of online recognition," Clin. Neurophysiol., vol. 111, pp. 477-481, 2000

[18] S. B. Wilson, C. A. Turner, R. G. Emerson, and M. L. Scheuer, "Spike detection II: Automatic, perception-based detection and clustering," Clin. Neurophysiol., vol. 110, pp. 404-411, 1999.

[19] S. J. Schiff, A. Aldroubi, M. Unser, and S. Sato, "Fast wavelet transformation of EEG," Electroenceph. Clin. Neurophysiol., vol. 91, pp. 442-455, 1994

[20] F. Sartorettoa and M. Ermani, "Automatic detection of epileptiform activity by single-level wavelet analysis," Clin. Neurophysiol., vol. 110, pp. 239-249, 1999.

[21] T. Kalayci and O. Ozdamar, "Wavelet preprocessing for automated neural network detection of EEG spikes," IEEE Eng. Med. Biol. Mag., vol. 14 , pp. 160-166, 1995.

[22] A. B. Geva and D. H. Kerem, "Forecasting generalized epileptic seizures from the EEG signal by wavelet analysis and dynamic unsupervised fuzzy clustering," IEEE Trans. Biomed. Eng., vol. 45, pp. 1205-1216, Oct. 1998.

[23] R. C. Eberhart, "Chaos theory for the biomedical engineer," IEEE Eng. Med. Biol. Mag., vol. BME-4, pp. 652-663, Sept. 1985.

[24] I. Yaylali, H. Kocak, and P. Jayakar, "Detection of seizures from small samples using nonlinear dynamic system theory," IEEE Trans. Biomed. Eng., vol. 43, pp. 743-751, July 1996.

[25] L. Diambra, J. C. Bastos de Figueiredo, and C. P. Malta, "Epileptic activity recognition in EEG recording," Physica A, vol. 273, pp. 495-505, 1999.

[26] J. Gotman, D. Flanagan, J. Shang, and B. Rosenblatt, "Automatic seizure detection in the newborn: Methods and initial evaluation," Electroenceph. Clin. Neurophysiol., vol. 103, no. 3, pp. 356-362, 1997.

[27] K. Arakawa, D. Fender, H. Harashima, H. Miyakawa, and Y. Saitoh, "Nonlinear digital filter for separating nonstationary waves from stationary waves of the EEG," in Proc. IEEE-IECEJ-ASJ Int. Conf. Acoustics, Speech, Signal Processing, 1986, pp. 1809-1812.

[28] S. S. Rao, Optimization: Theory and Application. New York: Wiley, 1984, pp. 231-233

[29] L. Zheng, D. T. Ye, F. S. Yang, and Y. T. Zhang, "Localization of hearing loss on the basis of transient-evoked otoacoustic emissions by using wavelet transform," in Proc. Conf. IEEE Engineering in Medicine and Biology Society, vol. 20, 1998, pp. 1531-1534.

[30] D. L. Jones and R. G. Baraniuk, "Efficient approximation of continuous wavelet transform," Electron. Lett., vol. 27, no. 9, pp. 748-750, 1991.
[31] R. A. Gopinath and C. S. Burrus, "Efficient computation of the wavelet transforms," in Proc. IEEE Conf. Acoustics Speech Signal Processing, vol. 3, 1990, pp. 1599-1601

[32] T. Zhang, F. S. Yang, and Q. Y. Tang, “A fast algorithm of continuous wavelet transform based on Mellin transform with biomedical application," in Proc. Conf. IEEE Engineering in Medicine and Biology Society, vol. 20, 1998, pp. 1142-1144.

[33] J. Bertrand, P. Bertrand, and J. P. Ovarlez, "Discrete Mellin transform for signal analysis," in Proc. IEEE Conf. Acoustics Speech Signal Processing, vol. 3, 1990, pp. 1603-1606.

[34] S. K. Pal and S. Mitra, "Multilayer perception, fuzzy sets and classification," IEEE Trans. Neural Networks, vol. 3, pp. 683-697, Oct. 1992.

[35] C. E. Metz, "Basic principles of ROC analysis," Sem. Nuclear Med., vol. 8, pp. 283-298, 1978.

[36] A. M. Beun, W. van Emde Boas, and E. Dekker, "Sharp transients in the sleep EEG of healthy adults: A possible pitfall in the diagnosis assessment of seizure disorders," Electroenceph. Clin. Neurophysiol., vol. 106, pp. 44-51, 1998.

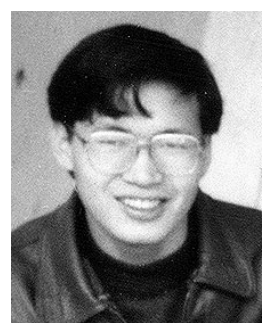

He Sheng Liu was born in Fukien, China, in 1975. He received the B.S. degree in biomedical engineering from Tsinghua University, Beijing, China, in 1997. He is currently working towards the $\mathrm{Ph} . \mathrm{D}$. degree in biomedical signal processing and pattern recognition at Tsinghua University.

His research interests include biomedical signal processing, brain-computer interface, epileptic EEG monitoring, EEG inverse problems, and machine learning/pattern recognition. Currently, he is working on brain-computer interface and spatio-temporal EEG pattern analysis.

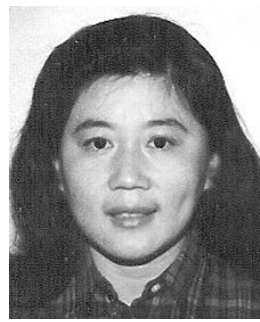

Tong Zhang received the B.S., M.S., and Ph.D. degrees in biomedical engineering from Tsinghua University, Beijing, China, in 1992, 1994, and 1996, respectively. She also received the M.S. and Ph.D. degrees in electrical engineering from the University of Southern California, Los Angeles, in 1998 and 1999, respectively.

Since February 2000, she has been working as a Research Scientist at the Imaging Technology Department of Hewlett-Packard Labs, Palo Alto, CA. Her research interest includes biomedical signal processing, multimedia content analysis, and audio/video database management. She authored the book Content-Based Audio Classification and Retrieval for Audiovisual Data Parsing (Norwell, MA: Kluwer, 2001).

Dr. Zhang served as Co-chair of the Conference on Internet Multimedia Management Systems III in SPIE's International Symposium ITCom, August 2002.

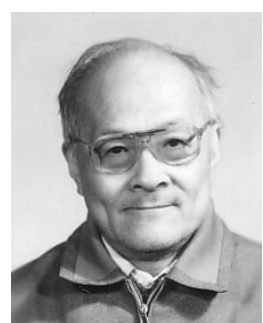

Fu Sheng Yang received the B.Sc. degree in electrical engineering from Amoy University, Fukien, China, in 1949.

Since 1951, he has been a Faculty Member of the Department of Electrical Engineering of Tsinghua University, Beijing, China, where he has held the rank of Professor since 1980. He is the author of Biomedical Signal Processing (China: Higher Education Press, 1989) and Engineering Analysis of Wavelet Transform and Its Application (China: Science Press, 1998). His current research interest lies mainly in the application of spatial analysis, time-frequency, time-scale analysis, and nonlinear dynamics analysis to biomedical signals. 